\title{
Erector spinae plane block for postoperative
} analgesia in patients undergoing total abdominal hysterectomy: a randomized controlled study original study

This article was published in the following Dove Press journal:

Journal of Pain Research

\author{
Mohamed Ahmed Hamed \\ Abeer Shaban Goda \\ Mohammed Magdy Basiony \\ Omar Sayed Fargaly \\ Mahdy Ahmed Abdelhady \\ Lecturer of Anesthesiology, Faculty of \\ Medicine, Fayoum University, Fayoum, \\ Egypt
}

Background: Abdominal hysterectomy is associated with marked postoperative pain and morbidity, but effective postoperative analgesia provides early recovery and ambulation.

Aim: We intended to assess the efficacy of bilateral erector spinae plane block (ESPB) on postoperative analgesia in females undergoing abdominal hysterectomy under general anesthesia.

Settings and Design: The design was a prospective, randomized, controlled, single-blind clinical study.

Patients and Methods: Sixty patients with American Society of Anesthesiologists (ASA) physical status classes I to III were scheduled for elective abdominal hysterectomy under general anesthesia, patients were randomly allocated into two equal groups. ESPB patients received ultrasound-guided ESPB at T9 vertebrae level with $20 \mathrm{ml}$ bupivacaine $0.5 \%$. Control group patients did not receive a block. Total fentanyl consumption in the first $24 \mathrm{~h}$ and visual analogue scale (VAS) score for pain were evaluated postoperatively. Unpaired Student's t-tests, chi-square tests, and Z tests were used to compare groups.

Results: No significant differences were recorded between the groups regarding age, weight, ASA physical status, or surgery duration, Total fentanyl consumption in the first $24 \mathrm{~h}$ was significantly higher in the control group than the ESPB group $(P=0.003 ; 485 \pm 20.39 \mathrm{mcg}$ vs $445 \pm 67.49 \mathrm{mcg}$, respectively), VAS for pain was significantly higher in the control group for the first $12 \mathrm{~h}$ postoperatively.

Conclusions: Bilateral ESPB provided effective postoperative analgesia and markedly decreased postoperative fentanyl consumption in patients undergoing an abdominal hysterectomy.

Keywords: erector spinae plane block, fentanyl consumption, total abdominal hysterectomy, postoperative analgesia

\section{Introduction}

Good postoperative analgesia can prevent morbidity associated with abdominal hysterectomy by allowing pain-free, early ambulation and decreasing the risks of long hospital stay, thromboembolism, and other poor outcomes. ${ }^{1}$ Epidural analgesia is commonly used in patients following abdominal hysterectomy except in cases with elevated intracranial tension, coagulopathy, patient refusal, and local sepsis. $^{2,3}$ Truncal blocks such as transversus abdominis plane (TAP) blocks have
Correspondence: Mohamed Ahmed Hamed

Department of Anesthesiology, Faculty of Medicine, Fayoum University, 635II,

province Fayoum, Egypt

Tel +200 I I I 8560065

Emailmah07@fayoum.edu.eg, 
seen limited success due to shorter duration and suboptimal analgesia. ${ }^{4}$ The ultrasound (US)-guided erector spinae plane block (ESPB) was initially described by Forero et al for providing thoracic analgesia at the $\mathrm{T} 5$ transverse process (TP). ${ }^{5}$ ESPB gained wide attention as it is a faster procedure that carries a lower risk of hypotension, can be used in patients with coagulopathy, is easy to perform, and requires less training. ${ }^{5}$ ESPB provides extensive, potent unilateral analgesia, is performed by local anesthetic injection in the plane between the erector spinae muscle and the TP. The local anesthetic diffuses into the paravertebral space through spaces between adjacent vertebrae and blocks both the dorsal and ventral branches of the thoracic spinal nerves. ${ }^{5,6}$ Bilateral ESPB performed at low thoracic levels was recently shown to provide satisfactory analgesia for gynecologic and abdominal surgery in case reports and series describing lower segment cesarean section, ventral hernia repair, and abdominoplasty. ${ }^{7-9}$

This study aimed to assess the efficacy of bilateral ESPB on postoperative analgesia in patients undergoing total abdominal hysterectomy under general anesthesia.

\section{Materials and methods}

This prospective, randomized, single-blind, controlled study was conducted in accordance with the Declaration of Helsinki. The study protocol was approved by the ethical committee of Fayoum University Hospital and registered at ClinicalTrials. gov (NCT03741348, no plan to share individual participant data (IPD)). Written informed consent was obtained from each patient before study enrolment. Prospective participants were scheduled for elective total abdominal hysterectomy via Pfanennstiel incision under general anesthesia between November 15, 2017 and October 15, 2018.

All women aged 40-70 years old who weighed $50-90 \mathrm{~kg}$, had an American Society of Anesthesiologists (ASA) physical status of classes I to III and were scheduled for elective total abdominal hysterectomy via Pfanennstiel incision under general anesthesia were included. Exclusion criteria were a local infection at the incision site; a history of hematological disorders including coagulation abnormality, previous abdominal surgeries, severe hepatic impairment, or chronic pain; or a known allergy to the study drugs.

An online randomization program was used to generate a random number list (1:1 ratio). Patient randomization numbers were concealed in opaque envelopes that were opened by the study investigator.

All members of the study group involved in obtaining functional data were blinded to randomization for the period of data acquisition and analysis. Patients were randomly allocated to two groups.

The ESPB group underwent US-guided ESPB at T9 vertebrae level with $20 \mathrm{ml}$ of bupivacaine $0.5 \%$. The control group underwent the same procedure but had a sham injection (20 ml of saline). One day before surgery, all patients were informed about the visual analogue scale (VAS), learned how to use patient-controlled analgesia (PCA) pump, and underwent routine investigations. In both groups, patients were monitored with electrocardiography, noninvasive blood pressure monitoring, and pulse oximetry. Intravenous (IV) access was established to administer IV midazolam at $0.01-0.02 \mathrm{mg} \cdot \mathrm{kg}^{-1}$. At the level of T9 and after skin sterilization, ESPB was administered in a sitting position. A linear US transducer (Phillips-Saronno Italy) was placed vertically $3 \mathrm{~cm}$ lateral to the midline to visualize back muscles: the trapezius above, the rhomboid major in the middle, and the erector-spinae muscle on the bottom, as well as the TPs with shimmering pleura in between. Next, 2-3 $\mathrm{ml}$ of $2 \%$ lidocaine was infiltrated. A 22-gauge short bevel needle (Spinocan, B. Braun Melsungen AG, Germany) was inserted in the cranial-caudal direction towards the TP in-plane with the US transducer until the needle touched the TP crossing all three muscles. Correct needle placement was confirmed by visualizing the needle in a plane along its entire length when the TP was contacted, and $1 \mathrm{ml}$ of anesthetic liquid was introduced. Hydrodissection of the interfascial plane between the erector spinae muscle and TP was confirmed by visualizing the local anesthetic spreading in a linear pattern between the muscle and the bony acoustic shadows of the TP. Then, up to $20 \mathrm{ml}$ bupivacaine $0.5 \%$ was injected. The same procedure was repeated on the other side of the back. The control group underwent the same procedure but had a sham injection ( $20 \mathrm{ml}$ saline).

Block success was tested by reduced cold sensation at the planned surgical incision site 5 min after block completion. Anesthesia was induced with fentanyl 2 mcg. $\mathrm{kg}^{-1}$ and propofol $2 \mathrm{mg} \cdot \mathrm{kg}^{-1}$ followed by atracurium $0.5 \mathrm{mg} \cdot \mathrm{kg}^{-1}$. After intubation, anesthesia was maintained with isoflurane (1 MAC) and atracurium $0.1 \mathrm{mg} \cdot \mathrm{kg}^{-1}$ as a maintenance dose every 30 min until the end of the procedure. After emerging from anesthesia, patients were transferred to the postanesthesia care unit (PACU) for a 2-h observation period. Patients were discharged from the PACU when they achieved a modified Aldrete score $\geq 9 .^{10}$

Postoperative analgesia was provided in both groups immediately after surgery by PCA fentanyl pump then oral acetaminophen $1 \mathrm{~g}$ four times a day. The criteria to stop the 
Table I Patient characteristics

\begin{tabular}{|l|l|l|l|}
\hline Variables & Control group, $\mathbf{n = 3 0}$ & ESPB group, $\mathbf{n = 3 0}$ & $\mathbf{p}$-Value \\
\hline Age & $50.7 \pm 4.72$ & $50.00 \pm 5.7$ & 0.607 \\
\hline Weight & $75.83 \pm 9.75$ & $73.17 \pm 10.63$ & 0.316 \\
\hline $\begin{array}{l}\text { ASA } \\
\text { I }\end{array}$ & $14(46.7 \%)$ & & $13(43.3 \%)$ \\
II & $12(40 \%)$ & $14(46.7 \%)$ & 0.846 \\
III & $4(13.3 \%)$ & $3(10.0 \%)$ & \\
\hline $\begin{array}{l}\text { Hysterectomy indication } \\
\text { Dysfunctional uterine bleeding }\end{array}$ & $17(28.3 \%)$ & $21(35.3 \%)$ & $9(15 \%)$ \\
Fibroid & $13(21.7 \%)$ & $89.83 \pm 19.36$ & 0.42 \\
\hline Surgery duration (min) & $91.17 \pm 20.87$ & & 0.798 \\
\hline
\end{tabular}

Note: All variables are reported as mean \pm SD.

Abbreviations: ASA, American Society of Anesthesiologists; ESPB, erector spinae plane block.

fentanyl titration protocol included satisfactory pain control, increased sedation (Ramsay sedation scale $>2$ ), decreased respiratory rate $<12 / \mathrm{min}$, decreased oxygen saturation $<95 \%$, or development of serious adverse effects (allergy, severe vomiting, hypotension). Self-reported VAS scores (ranging from 0 indicating no pain to 10 indicating extreme pain) were recorded $30 \mathrm{~min}$ and $2,4,6,12$, and $24 \mathrm{~h}$ postoperatively.

Any side effects such as nausea and vomiting and complications including pneumothorax were noted. Hospital stay duration from the first postoperative day to discharge was recorded.

The primary outcome of this study was total fentanyl consumption in the first $24 \mathrm{~h}$, and the secondary outcomes were VAS pain score (ranging from 0 indicating no pain to 10 indicating extreme pain) at several postoperative time points, hospital stay duration, and any complications.

\section{Statistical analysis}

The required sample size was calculated using the G power program 3.1.9.2. At least 10 patients were needed to have a power of least $80 \%$, with a two-sided $\alpha$ error of $5 \%$ level. Based on our previous studies, we would expect a difference in total fentanyl consumption in the first $24 \mathrm{~h}$ of $\sim 30 \%$ between the ESPB and control groups. The effect size was 0.735 . We calculated that 24 females would be required per treatment group. We included 30 per group to compensate for excluded patients.

Data were analyzed using IBM SPSS advanced statistics, version 21 (IBM Corp., Armonk, NY, USA).
Quantitative data are presented as mean \pm standard deviation, and qualitative data are presented as numbers and percentages.

Quantitative data were analyzed with unpaired student t-tests; qualitative data were analyzed using chi-square and $\mathrm{Z}$ tests. $P<0.05$ and $P<0.01$ was considered significant and highly significant, respectively.

\section{Results}

This study included 60 female patients (30 per group); no patients were excluded from the study. No significant between-group differences were noted for age, weight, ASA physical status, or surgery duration (Table 1). Total fentanyl consumption in the first $24 \mathrm{~h}$ was significantly higher in the control group compared to the ESPB group $(485 \pm 20.39 \mathrm{mcg}$ vs $445 \pm 67.49 \mathrm{mcg}$, respectively; $P=0.003$; Table 2). The VAS pain score was significantly higher in the control group for the first $12 \mathrm{~h}$ postoperatively (Table 3). No significant between-group differences were recorded regarding the duration of postoperative hospital stay (Table 4). No side effects or complications were recorded in either group.

Table 2 Total fentanyl consumption in the first $24 \mathrm{~h}$ after surgery

\begin{tabular}{|l|l|l|l|}
\hline Variable & $\begin{array}{l}\text { Control } \\
\text { group, } \mathbf{n = 3 0}\end{array}$ & $\begin{array}{l}\text { ESPB } \\
\text { group, } \mathbf{n = 3 0}\end{array}$ & $\mathbf{p}$-Value \\
\hline $\begin{array}{l}\text { Fentanyl } \\
\text { consumption }\end{array}$ & $485 \pm 20.39$ & $445 \pm 67.49$ & $0.003 *$ \\
\hline
\end{tabular}

Notes: All variables are reported as mean \pm SD. *statistically significant. Abbreviation: ESPB, erector spinae plane block. 
Table 3 Postoperative VAS score

\begin{tabular}{|l|l|l|l|}
\hline Time & $\begin{array}{l}\text { Control group, } \\
\mathbf{n = 3 0}\end{array}$ & $\begin{array}{l}\text { ESPB group, } \\
\mathbf{n = 3 0}\end{array}$ & $\boldsymbol{p}$-Value \\
\hline 30 minutes & $5.0 \pm 0.90$ & $3.3 \pm 1.74$ & $0.000^{*}$ \\
2 hours & $5.0 \pm 0.64$ & $3.2 \pm 1.82$ & $0.000^{*}$ \\
4 hours & $5.0 \pm 0.90$ & $3.3 \pm 1.74$ & $0.000^{*}$ \\
6 hours & $5.4 \pm 0.81$ & $3.3 \pm 1.82$ & $0.000^{*}$ \\
12 hours & $5.2 \pm 0.76$ & $4.5 \pm 1.54$ & $0.004^{*}$ \\
24 hours & $4.4 \pm 1.03$ & $4.6 \pm 1.52$ & 0.555 \\
\hline
\end{tabular}

Notes: All variables are reported as mean \pm SD. *statistically significant.

Abbreviations: ESPB, erector spinae plane block; VAS score, visual analogue scale score.

Table 4 Postoperative hospital stay duration

\begin{tabular}{|l|l|l|l|}
\hline Variable & $\begin{array}{l}\text { Control } \\
\text { group, } \mathbf{n}=\mathbf{3 0}\end{array}$ & $\begin{array}{l}\text { ESPB group, } \\
\mathbf{n}=\mathbf{3 0}\end{array}$ & $\mathbf{p}$-Value \\
\hline $\begin{array}{l}\text { Hospital stay } \\
\text { (days) }\end{array}$ & $2.4 \pm 0.49$ & $2.3 \pm 0.46$ & 0.425 \\
\hline
\end{tabular}

Note: All variables are reported as mean \pm SD.

Abbreviations: ESPB, erector spinae plane block.

\section{Discussion}

Patients usually experience significant pain after abdominal surgery, with the primary source of pain being the anterior abdominal wall and abdominal viscera. ${ }^{2}$ The ESPB was recently described as a way to provide analgesia through a truncal nerve block. ${ }^{5}$

Initially, the ESPB was defined at the level of the TP of $\mathrm{T} 5$, achieving an anesthetic distribution ranging from $\mathrm{C} 7-\mathrm{T} 1$ to T8 and resulting in effective analgesia of the ipsilateral thoracic wall. ${ }^{5}$ Because the erector spinae muscle extends throughout the lumbar region, an ESPB can also provide abdominal analgesia if it is performed at a lower level. ${ }^{11}$

The ESPB represents a more straightforward, safer alternative to epidural anesthesia because the ultrasonic target (TP) is easily visualized; the injection point is far from the neuroaxis, pleura, and large vascular structures; and wide craniocaudal diffusion of the anesthetic allows extensive coverage with a single injection. ${ }^{12}$ Initially described for thoracic analgesia, the ESPB was first used for thoracic pain management, ${ }^{5}$ costal fractures, ${ }^{13}$ breast surgeries, ${ }^{14}$ and as a technique for thoracoscopic lobectomy. ${ }^{15}$ There were also some publications describing its use for abdominal surgery, such as repair of ventral hernias, ${ }^{7}$ abdominoplasty, ${ }^{8}$ bariatric surgeries, ${ }^{16}$ laparoscopic abdominal surgeries, ${ }^{17}$ and lower segment cesarean section. ${ }^{9}$

ESPB can be performed at T4-5 levels for breast and thoracic surgeries and T7-8 levels for abdominal surgeries. ${ }^{7,18}$ A cadaver model demonstrated that when $20 \mathrm{ml}$ of fluid was injected at the T7 TP, it spread to the level of the C7-T 2 vertebra levels cranially and L2-3 vertebra levels caudally. ${ }^{7}$ Based on those publications, we decided to perform the blockade at the level of T9.

The current study was conducted to assess the postoperative analgesia efficacy of bilateral ESPB in females undergoing abdominal hysterectomy via Pfanennstiel incision under general anesthesia. Compared to patients without ESPB, there was a marked decrease in fentanyl consumption in the first $24 \mathrm{~h}$ after surgery.

Despite being one of the mainstays of postoperative pain management, opioid analgesic use is limited due to side effects like vomiting, sedation urinary retention, ileus, constipation, and respiratory depression. These side effects contribute to delayed hospital discharge and delayed resumption of normal activities of daily living. ${ }^{19}$ Moreover, opioid-induced immunosuppression can affect surgery outcomes including increased risks of infection and possibly metastasis in cancer patients. ${ }^{20}$ As a result, multimodal opioid-sparing analgesia has become an alternative for postoperative pain management. ${ }^{21}$ In our study, ESPB significantly reduced postoperative opioid consumption, indicating that it can be a component of multimodal opioid-sparing analgesia protocols.

Postoperative pain management is a major concern for both clinicians and patients. In the current study, the duration of analgesia was $>12 \mathrm{~h}$ for the ESPB group. VAS pain scores were significantly higher in the control group in the first $12 \mathrm{~h}$ postoperatively. Tulgar et $\mathrm{al}^{17}$ performed ESPB in three patients who underwent multiple abdominal procedures in a single surgical session and reported analgesia times of 17,13 , and $16 \mathrm{~h}$ for those cases. In the current study, the VAS score difference was statistically insignificant at $24 \mathrm{~h}$; this can be attributed to the use of a single injection. Catheter placement and intermittent boluses injection can prolong the duration of analgesia and lower pain scores for longer times. These data demonstrate that ESPB is a successful technique for postoperative pain control.

Postoperative pain contributes to increased morbidity, impaired physical function and quality of life, slowed recovery, prolonged opioid use during and after hospitalization, and increased cost of care. Moreover, early postoperative pain can trigger persistent pain that may last for months after surgery. $^{22}$ The rate of chronic pain after hysterectomy is reported to range from $5 \%-32 \% .^{23}$ Based on extensive evidence that correlates high postoperative pain levels with an increased prevalence of chronic postsurgical pain, ${ }^{24}$ our finding that early VAS scores were significantly lower in the ESPB 
group indicate that ESPB may also reduce chronic pain, but this requires additional studies. It is important to prevent postsurgical pain, as the cost of treating chronic pain that develops from acute pain in a 30-year-old individual could be as much as $\$ 1$ million over their estimated lifetime. ${ }^{25}$

A Cochrane systematic review of 23 randomized, controlled trials compared the effects of local and regional anesthetics with those of conventional analgesia in preventing chronic postoperative pain. Pooled results favored epidural anesthesia after thoracotomy and paravertebral block after breast cancer surgery for reducing the risk of pain at 6 months. ${ }^{26}$

The current study showed no difference in postoperative hospital stay duration, an outcome that is affected by numerous factors besides postoperative pain. No side effects or complications occurred in our study. Ueshima described only one complication after ESPB (pneumothorax). ${ }^{27}$

Limitations of this study include the short follow-up period and a lack of clinical studies that used this technique, which limited result comparisons. Despite these shortcomings, our findings demonstrate that ESPB has a role in decreasing opioid consumption and pain score and can used as a part of multimodal opioid-sparing analgesia protocol.

In conclusion, bilateral ESPB provided effective postoperative analgesia and markedly decreased postoperative fentanyl consumption in patients undergoing total abdominal hysterectomy.

\section{Disclosure}

The authors report no conflicts of interest in this work.

\section{References}

1. Azari L, Santoso JT, Osborne SE. Optimal pain management in total abdominal hysterectomy. Obstet Gynecol Surv. 2013;68:215-227. doi:10.1097/OGX.0b013e31827f5119

2. Carney J, McDonnell JG, Ochana A, Bhinder R, Laffey JG. The transversus abdominis plane block provides effective postoperative analgesia in patients undergoing total abdominal hysterectomy. Anesth Analg. 2008;107:2056-2060. doi:10.1213/ane.0b013e3181871313

3. Brull R, Macfarlane AJ, Chan VW. Spinal, epidural, and caudal anesthesia. In: Rd M, editor. Miller's Anaesthesia. 8thed. Vol. 1, Philadelphia (PA): Churchill Livingstone;2015:1684-1720.

4. Baeriswyl M, Kirkham KR, Kern C, Albrecht E. The analgesic efficacy of ultrasound-guided transversus abdominis plane block in adult patients: a meta-analysis. Anesth Analg. 2015;121:1640-1654. doi:10.1213/ANE.0000000000000967

5. Forero M, Adhikary SD, Lopez H, Tsui C, Chin KJ. The erector spinae plane block: a novel analgesic technique in thoracic neuropathic pain. Reg Anesth Pain Med. 2016;41:621-627. doi:10.1097/ AAP.0000000000000387
6. Ueshima H, Otake H. Similarities between the retrolaminar and erector spinae plane blocks. Reg Anesth Pain Med. 2017;42:123-124. doi:10.1097/AAP.0000000000000615

7. Chin KJ, Adhikary S, Sarwani N, Forero M. The analgesic efficacy of pre-operative bilateral erector spinae plane (ESP) blocks in patients having ventral hernia repair. Anaesthesia. 2017;72:452-460.

8. Ashok J, Priyanka J, Neelam S. The erector spinae block for postoperative analgesia in abdominoplasty - a case report. BAOJ Anesthesia. 2017;1:1.

9. Yamak Altinpulluk E, García Simón D, Fajardo-Pérez M. Erector spinae plane block for analgesia after lower segment caesarean section: case report. Rev Esp Anestesiol Reanim. 2018;65(5):284-286. doi:10.1016/j.redar.2017.11.006

10. Aldrete JA. The post anaesthesia recovery score revisited. J Clin Anesth. 1995;7:89-91.

11. Chin KJ, Forero M, Adhikary SD. Reply to Dr. Ueshima and Dr. Murouchi. Reg Anesth Pain Med. 2017;42:124-125. doi:10.1097/AAP.0000000000000615

12. Muñoz F, Cubillos J, Bonilla AJ, Chin KJ. Erector spinae plane block for postoperative analgesia in pediatric oncological thoracic surgery. Can J Anaesth. 2017;64:880-882. doi:10.1007/ s12630-017-0894-0

13. Hamilton DL, Manickam B. Erector spinae plane block for pain relief in rib fractures. Br J Anaesth. 2017;118:474-475. doi:10.1093/bja/ aew387

14. Bonvicini D, Giacomazzi A, Pizzirani E. Use of the ultrasound-guided erector spinae plane block in breast surgery. Minerva Anestesiol. 2017;83:1111-1112. doi:10.23736/S03759393.17.12141-3

15. Scimia P, Basso Ricci E, Droghetti A, Fusco P. The ultrasound-guided continuous erector spinae plane block for postoperative analgesia in video-assisted thoracoscopic lobectomy. Reg Anesth Pain Med. 2017;42:537. doi:10.1097/AAP.0000000000000615

16. Chin KJ, Malhas L, Perlas A. The erector spinae plane block provides visceral abdominal analgesia in bariatric surgery: a report of 3 cases. Reg Anesth Pain Med. 2017;42:372-376. doi:10.1097/ AAP.0000000000000615

17. Tulgar S, Selvi O, Kapakli MS. Erector spinae plane block for different laparoscopic abdominal surgeries: case series. Anesthesiology. 2018;2018:3947281.

18. Bonvicini D, Tagliapietra L, Giacomazzi A, Pizzirani E. Bilateral ultrasound-guided erector spinae plane blocks in breast cancer and reconstruction surgery. J Clin Anesth. 2018;44:3-4. doi:10.1016/j. jclinane.2017.10.006

19. White PF. The changing role of non-opioid analgesic techniques in the management of postoperative pain. Anesth Analg. 2005;101:S5S22. doi:10.1213/01.ANE.0000177099.28914.A7

20. Sacerdote P, Franchi S, Panerai AE. Non-analgesic effects of opioids: mechanisms and potential clinical relevance of opioid-induced immunodepression. Curr Pharm Des. 2012;18(37):6034-6042. doi: $10.2174 / 138161212803582496$

21. Rafiq S, Steinbruchel DA, Wanscher MJ, et al. Multimodal analgesia versus traditional opioid based analgesia after cardiac surgery, a randomized controlled trial. J Cardiothoracic Surg. 2014;9(52):1-8.

22. Gan TJ. Poorly controlled postoperative pain: prevalence, consequences, and prevention. J Pain Res. 2017;10:2287-2298. doi:10.2147/JPR.S134133

23. Theunissen M, Peters ML, Schepers J, et al. Recovery 3 and 12 months after hysterectomy: epidemiology and predictors of chronic pain, physical functioning, and global surgical recovery. Medicine (Baltimore). 2016;95(26):e3980. doi:10.1097/ MD.0000000000004864 
24. Brandsborg B, Dueholm M, Nikolajsen L, Kehlet H, Jensen TS. A prospective study of risk factors for pain persisting 4 months after hysterectomy. Clin J Pain. 2009;25(4):263-268. doi:10.1097/ AJP.0b013e31819655ca

25. Cousins MJ, Power I, Smith G. Pain: a persistent problem. Reg Anesth Pain Med. 2000;25:6-21.
26. Andreae MH, Andreae DA. Local anaesthetics and regional anaesthesia for preventing chronic pain after surgery. Cochrane Database Syst Rev. 2012;10:CD007105.

27. Ueshima H. Pneumothorax after the erector spinae plane block. $J$ Clin Anesth. 2018;48:12. doi:10.1016/j.jclinane.2018.04. 009

\section{Publish your work in this journal}

The Journal of Pain Research is an international, peer reviewed, open access, online journal that welcomes laboratory and clinical findings in the fields of pain research and the prevention and management of pain. Original research, reviews, symposium reports, hypothesis formation and commentaries are all considered for publication. The manuscript management system is completely online and includes a very quick and fair peer-review system, which is all easy to use. Visit http:// www.dovepress.com/testimonials.php to read real quotes from published authors. 